\title{
Outpatient antibiotic therapy and short term mortality in elderly patients with chronic obstructive pulmonary disease
}

\author{
Don D Sin MD MPH ${ }^{1,3}$, Jack V Tu MD PhD ${ }^{1,2}$ \\ ${ }^{1}$ Institute for Clinical Evaluative Sciences and ${ }^{2}$ Department of Medicine, Sunnybrook and \\ Women's College Health Science Centre, University of Toronto, Toronto, Ontario; ${ }^{3}$ Department \\ of Medicine, University of Alberta, Edmonton, Alberta
}

DD Sin, JV Tu. Outpatient antibiotic therapy and short term mortality in elderly patients with chronic obstructive pulmonary disease. Can Respir J 2000;7(6):466-471.

OBJECTIVE: To determine the association between outpatient use of oral antibiotics and 30-day all-cause mortality following hospitalization in a group of elderly chronic obstructive pulmonary disease (COPD) patients.

DESIGN: A population-based retrospective cohort study.

SETTING: Ontario, Canada.

POPULATION STUDIED: All 26,301 patients, 65 years of age or older, who were hospitalized for COPD between 1992 and 1996 in Ontario.

METHODS: All elderly patients admitted at least once with a most responsible diagnosis of COPD using the Canadian Institute for Health Information database were identified. They were then linked to the Ontario Drug Benefit database to determine the use of antibiotics within 30 days of the index hospitalization and to the Ontario registered persons database to determine the 30-day mortality following their index hospitalization.

RESULTS: Outpatient use of antibiotics within 30 days before the index hospitalization was associated with a significant reduction in the 30-day mortality following hospitalization (odds ratio [OR] $0.83,95 \%$ CI 0.75 to 0.92 ). Use of macrolides had the lowest relative odds for mortality (OR $0.58,95 \%$ CI 0.47 to 0.73 ), while use of fluoroquinolones had the highest relative odds (OR $0.98,95 \%$ CI 0.84 to 1.15 ). CONCLUSIONS: Use of antibiotics before hospitalization was associated with a significant reduction in the risk of short term mortality among a group of elderly COPD patients who eventually required hospitalization for their disease. These findings support the early use of antibiotics in COPD patients who experience an acute exacerbation.

Key Words: Antibiotics; Chronic obstructive pulmonary disease; Mortality

\section{Antibiothérapie en clinique externe et mortalité à court terme chez des patients âgés souffrant de BPOC}

OBJECTIF : Déterminer quel est le lien entre l'administration d'antibiotiques oraux en clinique externe et la mortalité de toutes causes dans les 30 jours suivant une hospitalisation chez un groupe de patients âgés souffrant de BPOC (bronchopneumopathie obstructive chronique).

MODÈLE : Étude de cohorte rétrospective basée dans la population.

voir page suivante

This work was supported in part by the Institute for Clinical Evaluative Sciences, which is funded by the Ontario Ministry of Health. The results and conclusions are strictly those of the authors and should not be attributed to any of the sponsoring agencies

Dr Sin was supported by a fellowship from the Alberta Heritage Foundation for Medical Research at the time of this work; Dr Tu is supported by a Medical Research Council of Canada Scholarship Award

Correspondence and reprints: Dr Don D Sin, 2 E4.39 Walter C Mackenzie Health Sciences Centre, University of Alberta, Edmonton, Alberta T6G 2B7. Telephone 780-407-6151, fax 780-407-6384, e-mail don.sin@ualberta.ca 
CONTEXTE : Ontario, Canada.

POPULATION ÉTUDIÉE : Les 26301 patients de 65 ans ou plus qui ont été hospitalisés pour BPOC entre 1992 et 1996 en Ontario.

MÉTHODES : Tous les sujets âgés admis au moins une fois pour un diagnostic probable de BPOC selon la base de données de l'Institut canadien d'information sur la santé ont été identifiés. Ils ont ensuite été reliés à la base de données du Régime de médicaments gratuits de l'Ontario afin de quantifier l'utilisation d'antibiotiques dans les 30 jours de l'hospitalisation initiale avant d'être reliés à la base de données des personnes inscrites en Ontario afin de déterminer le taux de mortalité à 30 jours suivant l'hospitalisation initiale.

RÉSULTATS : L'administration en clinique externe d'une antibiothérapie dans les 30 jours précédant une hospitalisation initiale a été associée à une réduction significative de la mortalité dans les 30 jours suivant l'hospitalisation (risque relatif (RR) 0,83, IC $95 \%, 0,75$ à 0,92). L'emploi des macrolides a donné lieu au risque relatif de mortalité le plus bas (RR 0,58, IC $95 \%, 0,47$ à 0,73), alors que l'utilisation des fluoroquinolones a donné lieu au risque relatif le plus élevé (RR 0,98, IC $95 \%, 0,84$ à 1,15).

CONCLUSION : Le recours à l'antibiothérapie avant l'hospitalisation a été associé à une réduction significative du risque de mortalité à court terme dans un groupe de sujets âgés souffrant de BPOC qui ont éventuellement nécessité une hospitalisation pour leur maladie. Ces conclusions appuient l'utilisation précoce des antibiotiques chez des patients souffrant de BPOC qui présentent une poussée aiguë d'exacerbation.
$\mathrm{C}$ hronic obstructive pulmonary disease (COPD) is a leading cause of morbidity and mortality. Between 1980 and 1995, COPD-related mortality increased by $93 \%$, representing the largest increase in mortality among all major diseases in Canada (1). The natural history of COPD is characterized by frequent exacerbations resulting in increased cough, purulent sputum production and dyspnea (2). Although sputum cultures show no growth in approximately $50 \%$ of COPD exacerbations, bacterial infections are thought to be the leading cause of COPD flare-ups $(3,4)$. Antibiotic therapy may, therefore, modify the course of the disease (5). However, previous studies of antibiotic therapy during COPD exacerbations have produced inconsistent results. Several studies have failed to demonstrate a beneficial effect of oral antibiotic therapy (6-8), while others have shown improvements in lung function and patient symptoms $(9,10)$. One explanation for these differences is that previous studies have generally had small sample sizes and, therefore, may have lacked the statistical power to detect subtle but important beneficial effects of antibiotic therapy on the course of COPD exacerbations. Another possibility is that the effectiveness of antibiotic therapy may diminish if treatment is delayed. For example, a study by Nicotra and coworkers (8) failed to demonstrate a significant benefit of antibiotic therapy in COPD. However, in their group of patients, antibiotics were used after their patients were hospitalized (8). In contrast, Anthonisen and coworkers (9) used antibiotics on an outpatient basis and showed that antibiotic therapy produced better health outcomes than standard therapy alone.

To overcome the problems of small sample sizes and to determine the impact of antibiotics on mortality, we conducted a large population-based study of COPD patients who were hospitalized in Ontario for an exacerbation of their disease. We sought to determine the association between initial outpatient oral antibiotic therapy and 30-day mortality, and to describe the initial oral antibiotic therapy most frequently prescribed on an outpatient basis for COPD patients who were subsequently hospitalized for their disease.

\section{PATIENTS AND METHODS}

Study population: The Canadian Institute for Health Information hospital discharge database was used to identify all patients who were hospitalized with a "most responsible diagno- sis" of COPD between April 1, 1992 and March 31, 1997. COPD was identified using the International Classification of Diseases, ninth revision (ICD-9) codes 490, 491, 492 and 496. For those with multiple hospitalizations during the study period, only the initial hospitalization was used in the analysis in order to avoid double counting of patients. Patients who were transferred from a chronic care facility to an acute care hospital were excluded because outpatient drug information was not available for such patients. Patients younger than 65 years of age were also excluded because the databases did not contain any prescription medication information for this group of patients. Thus, the analysis contained only those COPD patients 65 years of age or older.

Medication information: During the study period, the Ontario government offered prescription medications free of charge to all residents 65 years of age or older through the Ontario Drug Benefit Program. The Ontario Drug Benefit database, therefore, contained comprehensive data on all outpatient medications including the name, the formulation and the amount that was dispensed to all patients in this cohort. From this database, the use of oral antibiotics within 30 days of the index hospitalization was determined. The medications selected were amoxicillin (or ampicillin), penicillin, sulfa drugs, cephalosporins, fluoroquinolones, tetracyclines and macrolides. These medications were chosen because previous studies have shown that they are the most widely used antibiotics for COPD exacerbations (10).

Mortality data: By law, all deaths occurring in Ontario must be reported to the provincial government. This information is then captured in the Ontario Registered Persons Database. The Registered Persons Database was used in the present study to determine the 14-day and 30-day mortality from the date of the index hospitalization for patients in this cohort. The primary analysis was conducted using the 30-day mortality rate as the outcome variable of interest.

The accuracy and reliability of these administrative databases in Ontario have been demonstrated previously $(11,12)$. Using a unique identifier contained in each of these data sets, the databases were linked together. To protect patient confidentiality, these identifiers were scrambled before crosslinkages took place.

Confounders: To control for the severity of COPD, patients' use of other anti-COPD medications, including beta2-agonists, 
TABLE 1

Baseline characteristics of chronic obstructive pulmonary disease (COPD) patients who did and did not receive outpatient antibiotics within $\mathbf{3 0}$ days of the index hospitalization

\begin{tabular}{lccc}
\hline Variable & $\begin{array}{c}\text { Antibiotic } \\
\text { user }\end{array}$ & $\begin{array}{c}\text { Antibiotic } \\
\text { nonuser }\end{array}$ & P \\
\hline Number of patients & 9037 & 17,264 & - \\
& $(34.4 \%)$ & $(66.6 \%)$ & \\
Age (years \pm SD) & $75.1 \pm 6.7$ & $75.5 \pm 6.8$ & 0.001 \\
Male (\%) & 53.7 & 57.2 & 0.001 \\
Charlson-Deyo score & $0(0,1)$ & $0(0,1)$ & 0.841 \\
$\quad$ (median), IQR & & & \\
Myocardial infarction (\%) & 3.4 & 3.4 & 0.993 \\
Congestive heart failure (\%) & 15.2 & 16.7 & 0.001 \\
Diabetes (\%) & 9.9 & 9.9 & 0.852 \\
Malignancy (\%) & 7.8 & 7.3 & 0.321 \\
Chest radiograph ${ }^{*}(\%)$ & 32.0 & 15.9 & 0.001 \\
Outpatient office visits ${ }^{*}(\%)$ & 79.0 & 54.2 & 0.001 \\
\hline
\end{tabular}

${ }^{*}$ Outpatient visits to physicians for COPD and use of chest radiograph within one year of the index hospitalization. IQR Interquartile range

ipratropium bromide, oral corticosteroids and oral theophylline derivatives, was determined. Categorical variables were created for each of these anti-COPD medications to indicate whether they were used by the patients in the cohort within 30 days of the index hospitalization. Use of various health services including outpatient physician visits for COPD within one year of hospitalization was also determined. To estimate the patient's burden of comorbidity, a modified CharlsonDeyo comorbidity score (13) was calculated for each patient using the 15 secondary diagnostic fields contained in the Canadian Institute for Health Information discharge database. To derive this score, the presence of the following comorbid conditions was taken into account: congestive heart failure (ICD-9 codes 428.x); ischemic heart disease (ICD-9 codes 410.x, 412.x); peripheral vascular disease (ICD-9 codes 443.9, 441.x, 785.4, V43.4); cerebrovascular accidents (ICD-9 codes 430.x-438.x); dementia (ICD-9 codes 290.x); rheumatological diseases (ICD-9 codes 710.0, 710.1, 710.4, 714.0-714.2, 714.81, 725.x); peptic ulcer disease (ICD-9 codes 531.x534.9); diabetes (ICD-9 codes 250.0-250.7); malignancy (ICD-9 codes 140.x-172.9, 174.x-195.8, 196.x-199.1, 200.x208.9); renal diseases (ICD-9 codes 582.x, 583.x, 585.x, 586.x, 588.x); and liver diseases (ICD-9 codes 571.x, 572.x) (14). A Charlson-Deyo comorbidity score of 0 denotes the absence of any comorbidities; a higher number indicates increasing burden of comorbidities. In the present study, the median Charlson-Deyo comorbidity score was 0 with a range of scores of 0 to 10 .

Statistical analyses: The means and standard deviations from continuous variables were compared using the twotailed Student's $t$ test, while binary variables were compared using a $\chi^{2}$ test for contingency tables. A comparison of the 30 -day mortality rate between patients receiving and not receiving outpatient antibiotic therapy was based on a multiple logistic regression model. Age, sex, Charlson-Deyo
TABLE 2

Use of various antichronic obstructive pulmonary disease (anti-COPD) medications by patients with COPD within 30 days of the index hospitalization among users and nonusers of antibiotics

\begin{tabular}{lccc}
\hline Variable & $\begin{array}{c}\text { Antibiotic } \\
\text { user }\end{array}$ & $\begin{array}{c}\text { Antibiotic } \\
\text { nonuser }\end{array}$ & $\mathbf{P}$ \\
\hline Number of patients & $9037(34.4 \%)$ & $17,264(66.6 \%)$ & - \\
Beta2-agonists (\%) & 59.9 & 44.9 & 0.001 \\
Anticholinergics (\%) & 35.0 & 27.4 & 0.001 \\
Oral steroids (\%) & 26.2 & 11.5 & 0.001 \\
Theophylline (\%) & 15.2 & 11.3 & 0.001 \\
\hline
\end{tabular}

comorbidity score, use of other anti-COPD medications and health services were included in the adjusted model as covariates. From this model, the relative odds ratios (ORs) and associated $95 \%$ CIs were calculated using standard methods (15). All tests were two-tailed, and $\mathrm{P}<0.05$ was considered statistically significant. As a part of sensitivity analyses, the same set of analyses using were conducted 14-day mortality as the outcome of interest. All analyses were conducted using SAS version 6.12 (SAS Institute Inc, United States).

\section{RESULTS}

Demographics of the study population: In total, 26,301 patients were included in the study; $7 \%(\mathrm{n}=1937)$ of patients died within 30 days of hospitalization. Overall, $34.4 \%$ $(n=9037)$ of the patients used an oral antibiotic within 30 days of their index hospitalization date. The baseline characteristics of users and nonusers of outpatient antibiotics are described in Table 1. Female patients were more likely to use an antibiotic before hospitalization than male patients (OR 1.15, $95 \%$ CI 1.09 to 1.21 ). Patients who were 80 years of age or older had the highest rate of antibiotic use (30\%), while those between 65 and 70 years of age had the lowest rate of use (22\%). The Charlson-Deyo comorbidity scores were similar between the two groups.

Use of other anti-COPD medications and health services: Patients exposed to outpatient antibiotic therapy were more likely to have received other anti-COPD medications within 30 days of hospitalization (Tables 1 and 2). Those who received antibiotics were more likely to use inhaled beta2-agonists (OR $1.83,95 \%$ CI 1.74 to 1.93 ), ipratropium bromide (OR $1.43,95 \%$ CI 1.35 to 1.51 ), oral corticosteroids (OR 2.73, 95\% CI 2.56 to 2.92) and oral theophylline derivatives (OR $1.41,95 \%$ CI 1.31 to 1.52 ) than those who did not use antibiotics as an outpatient. Patients who were treated with an antibiotic medication were also more likely to have had an office visit to a physician within one year of their index hospitalization date (OR 3.18, $95 \%$ CI 3.00 to 3.37 ).

Temporal trends in the use of antimicrobial medications for patients in the study: Oral cephalosporins were the most popular antibiotics used for COPD exacerbations in Ontario. These medications were used by $27 \%$ of patients who received outpatient antibiotic therapy. Figure 1 shows the prevalence of use of various antibiotics between 1992 and 


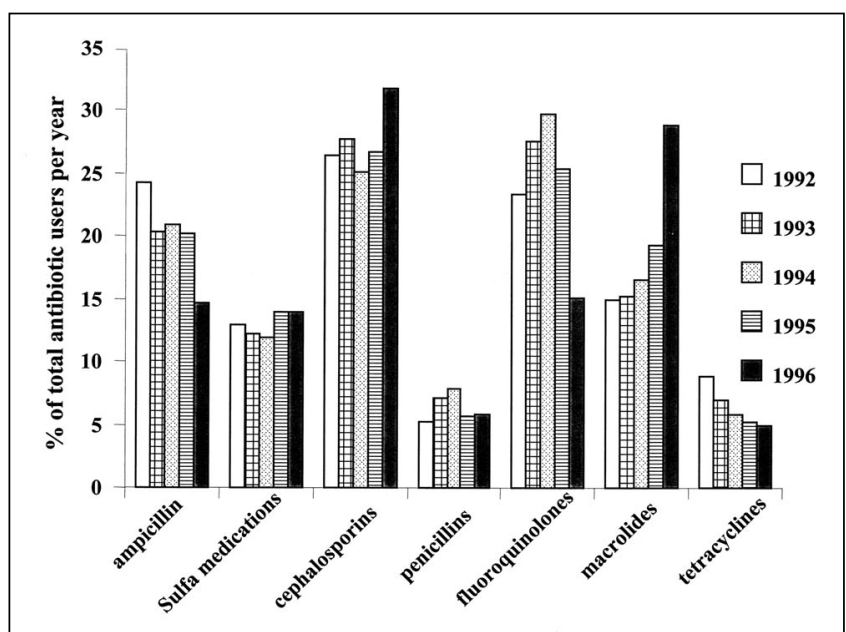

Figure 1) Temporal changes in the frequency of use of various antibiotics among chronic obstructive pulmonary disease patients in the study who received at least one antibiotic before hospitalization between 1992 and 1996

1996. The popularity of the conventional oral antibiotics such as penicillin, amoxicillin (or ampicillin), tetracyclines and sulfa-based drugs has declined over the years, while macrolides have experienced the biggest increase in use. The steep increase in macrolide use between 1994 and 1996 can be attributed almost entirely to the introduction of clarithromycin into the market. In 1995 , only $6.7 \%$ of the patients who used antibiotics received clarithromycin. By 1996, 18.4\% of patients were using clarithromycin. At the same time, there was a proportional decrease in the use of fluoroquinolones in the population.

Association between the use of antimicrobial medications and mortality: Patients who used antibiotics within 30 days of the index hospitalization date experienced lower odds for all-cause 30-day mortality after hospitalization than those who did not receive antibiotics (OR $0.86,95 \%$ CI 0.78 to $0.95)$. Other variables that were significantly associated with 30-day mortality were age (OR $1.05,95 \%$ CI 1.04 to 1.06), Charlson-Deyo comorbidity score (OR 1.30, 95\% CI 1.26 to 1.35 ) and male sex (OR $1.31,95 \%$ CI 1.19 to 1.44 ). When adjustments were made for important baseline covariates including age, sex, Charlson-Deyo comorbidity scores and use of other anti-COPD medications, the adjusted relative odds for the 30-day mortality associated with antibiotic use was 0.83 (95\% CI 0.75 to 0.92 ). Table 3 describes the association between various variables and the adjusted risk of death within 30 days of hospitalization. When 14-day mortality was considered, the relative odds associated with antibiotic exposure before hospitalization was 0.79 (95\% CI 0.70 to 0.90 ). The direction of the individual relative odds estimates for the 30-day mortality associated with each class of antibiotic was similar, with all of the antibiotics showing a protective effect against all-cause mortality (Figure 2). Of these, macrolides had the lowest relative odds for mortality (OR 0.58 ; $95 \mathrm{CI} 0.47$ to 0.73 ), while fluoroquinolones had the highest relative odds (OR $0.98,95 \%$ CI 0.84 to 1.15$)$.
TABLE 3

Adjusted odds ratios of variables associated with all-cause mortality among patients with chronic obstructive pulmonary disease within $\mathbf{3 0}$ days of the index hospitalization*

\begin{tabular}{lccc}
\hline Variable & Odds ratio & $\mathbf{9 5 \%} \mathbf{C l}$ & $\mathbf{P}$ \\
\hline Age & 1.05 & 1.05 to 1.06 & 0.001 \\
Charlson-Deyo score $^{\dagger}$ & 1.30 & 1.26 to 1.34 & 0.001 \\
Male $^{\ddagger}$ & 1.23 & 1.17 to 1.36 & 0.001 \\
$\begin{array}{l}\text { Use of various medications within } 30 \text { days of the } \\
\text { index hospitalization }\end{array}$ & & & \\
Antibiotics & 0.83 & 0.75 to 0.92 & 0.001 \\
Anticholinergics & 1.38 & 1.25 to 1.53 & 0.001 \\
Oral corticosteroids & 1.44 & 1.28 to 1.63 & 0.001 \\
Theophyllines & 1.28 & 1.12 to 1.46 & 0.001 \\
\hline
\end{tabular}

*The final model was adjusted for all of the above variables and use of physician's services; ${ }^{\dagger}$ Reference category was a Charlson-Deyo score of $O$ (ie, no comorbidity) and the odds ratio indicates a 1-unit increase in the Charlson-Deyo score; ${ }^{\ddagger}$ Reference category is elderly female patients with chronic obstructive pulmonary disease; ${ }^{\S}$ Reference category was nonuse of these medications

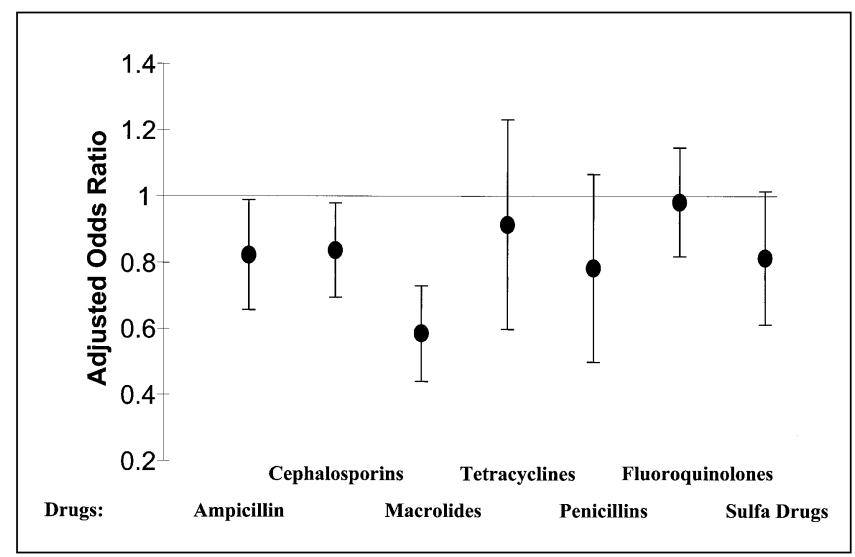

Figure 2) Adjusted odds ratios comparing the use of various antibiotics prehospitalization and 30-day mortality. The reference group comprised the patients who did not receive any antibiotics before hospitalization. The odds ratios were adjusted for age, sex, comorbidity and use of antichronic obstructive pulmonary disease medications. The bars represent $95 \%$ CIs

\section{DISCUSSION}

In a cohort of elderly patients with COPD who required hospitalization for their COPD, we found that treatment with oral antibiotics on an outpatient basis before their COPD admission was associated with a reduced mortality rate. COPD patients who received at least one course of antibiotic therapy before hospitalization were $17 \%$ less likely to die within 30 days following hospitalization for their COPD than those who did not receive any antibiotic therapy. These differences persisted even after adjustment for various confounding factors such as age, sex and use of other anti-COPD medications. These findings are consistent with previous studies on the effect of early antibiotic therapy on the health outcomes of patients with respiratory infections, which showed that antibiotic therapy in COPD hastens recovery and improves patient symptoms $(9,10)$. We extend these findings by demonstrating that outpatient antibiotic therapy for severe COPD exacerba- 
tions may also reduce all-cause mortality in patients who eventually require hospitalization for their disease.

Although in over $50 \%$ of the cases the inciting bacterial organism is never identified (5), empirical antibiotic therapy improves airway inflammation, resulting in enhanced mucociliary clearance, and reduced patient symptoms including cough (14) and mucous production (16). In contrast, inadequate antimicrobial coverage during COPD exacerbations may facilitate bacterial spread through the bronchial tree and stimulate an inflammatory response, which may result in mucosal damage and make the patient more vulnerable to progressive deterioration in respiratory function (17). Because it may be difficult to separate viral from bacterial infections of the airway on a clinical basis, our findings support the empirical use of antibiotic therapy for COPD exacerbations to reduce airway inflammation and enhance their overall survival from their acute illness.

Of the antibiotics in our study, use of macrolides was associated with the highest relative risk reduction, while fluoroquinolones demonstrated the lowest relative risk reduction. There is growing concern that some fluoroquinolones, particularly the older generation, do not have consistent coverage against Streptococcus pneumoniae (18), which is felt to be a leading pathogen in COPD exacerbations and the organism associated with the highest level of airway inflammation and morbidity (19). An alternate explanation is that the use of fluoroquinolones in our study was a marker for more severe COPD exacerbations (20). Because leading experts have recommended fluoroquinolones as second- or third-line antibiotics for COPD exacerbations (21), community physicians may reserve them for COPD patients who are refractory to conventional antibiotics.

The present study has several limitations that deserve further discussion. First, our study was observational in nature and, therefore, antimicrobial treatment selection biases may have been present. To minimize these biases, we controlled for potential confounding by age, sex, comorbidity and severity of illness as measured by the use of various anti-COPD medications and health service utilization before their index hospitalization. Even with these adjustments, we found a difference in the 30-day mortality rate between those treated and not treated with antibiotic therapy before hospitalization, suggesting that selection biases did not account for our findings. Second, the case definition of antibiotic user was on the basis of patients' exposure to an antibiotic within 30 days of their index hospitalization. Because we did not have information on patient compliance, it is possible that some patients who received these medications did not actually take them. However, this type of misclassification in exposure status would have led to an underestimation of the magnitude of association between antibiotic use and mortality but would not change the overall direction of the relationship (21). Third, we did not have any information on those COPD patients who were treated with antibiotics for their exacerbation but who were not admitted to an acute care hospital. If outpatient antibiotic therapy resulted in an excess mortality before hospitalization compared with no therapy, the exclu- sion of these patients could have confounded our findings. However, outpatient antibiotic use is generally well tolerated by COPD patients and severe complications leading to hospitalization or mortality have been rarely reported (5). Thus, it is unlikely that such a bias could explain our findings. Fourth, we did not have any information on inpatient treatment of these patients. Our findings assumed that patients who received and did not receive antibiotic therapy on an outpatient basis received similar care while in hospital. Our databases do not contain any information concerning in-hospital care, which is an important limitation to our study.

Finally, confounding by indication needs to be considered carefully. Was the use of antibiotics just a marker for patients who already had a better prognosis than nonusers? This hypothesis is unlikely based on several lines of evidence. First, the use of antibiotics is still controversial in COPD management. Therefore, a consensus on the type of patients who respond optimally to these drugs does not exist (2), which makes it unlikely that patients with a good prognosis would be preferentially treated with antibiotics. Second, the baseline demographic features were similar between users and nonusers of antibiotic therapy. Third, antibiotic users were more likely to have used anti-COPD medications and other health services, including outpatient physicians' offices, for their COPD than nonusers of antibiotics. Because these measures are surrogate markers for COPD severity (23), users of antibiotics were more likely to have greater COPD severity than nonusers of antibiotics.

\section{CONCLUSIONS}

This study of COPD patients living in the community demonstrated that outpatient use of antibiotics was associated with significantly lower 14-day and 30-day mortality risks than those who were not treated with antibiotics. These findings, therefore, suggest that aggressive outpatient management of COPD patients with antibiotic therapy during exacerbations may reduce mortality in these patients. However, before this recommendation is implemented widely, carefully conducted randomized, controlled trials are required to confirm these initial findings.

\section{REFERENCES}

1. Lacasse Y, Brooks D, Goldstein RS. Trends in the epidemiology of COPD in Canada, 1980 to 1995. COPD and Rehabilitation Committee of the Canadian Thoracic Society. Chest 1999;116:306-13.

2. Ferguson GT, Cherniack RM. Management of chronic obstructive pulmonary disease. N Engl J Med 1993;328:1017-22.

3. Soler N, Torres A, Ewig S, et al. Bronchial microbial patterns in severe exacerbations of chronic obstructive pulmonary disease (COPD) requiring mechanical ventilation. Am J Respir Crit Care Med 1998;157:1498-505.

4. Miravitlles M, Espinosa C, Fernández-Laso E, Martos JA, Maldonado JA, Gallego M. Relationship between bacterial flora in sputum and functional impairment in patients with acute exacerbations of COPD. Study Group of Bacterial Infection in COPD. Chest 1999;116:40-6.

5. Grossman RF. The value of antibiotics and the outcomes of antibiotic therapy in exacerbations of COPD. Chest 1998;113(Suppl 4):249S-55S.

6. Jorgensen AF, Coolidge J, Pedersen PA, Petersen KP, Waldorff S, Widding E. Amoxicillin in treatment of acute uncomplicated exacerbations of chronic bronchitis. A double-blind, placebo-controlled 
multicentre study in general practice. Scand J Prim Health Care 1992;10:7-11.

7. Sachs AP, Koeter GH, Groenier KH, van der Waaij D, Schiphuis J, Meyboom-de Jong B. Changes in symptoms, peak expiratory flow, and sputum flora during treatment with antibiotics of exacerbations in patients with chronic obstructive pulmonary disease in general practice. Thorax 1995:50:758-63.

8. Nicotra MB, Rivera M, Awe RJ. Antibiotic therapy of acute exacerbations of chronic bronchitis. A controlled study using tetracycline. Ann Intern Med 1982;97:18-21.

9. Anthonisen NR, Manfreda J, Warren CPW, Hershfield ES, Harding GKM, Nelson NA. Antibiotic therapy in exacerbations of chronic obstructive pulmonary disease. Ann Intern Med 1987;106:196-204

10. Saint S, Bent S, Vittinghoff E, Grady D. Antibiotics in chronic obstructive pulmonary disease exacerbations. A meta-analysis. JAMA 1995;273:957-60.

11. Redelmeier DA, Tan SH, Booth GL. The treatment of unrelated disorders in patients with chronic medical diseases. N Engl J Med 1998;338:1516-20.

12. Tu JV, Hannan EL, Anderson GM, et al. The fall and rise of carotid endarterectomy in the United States and Canada. N Engl J Med 1998;339:1441-7.

13. Deyo RA, Cherkin DC, Ciol MA. Adapting a clinical comorbidity index for use with ICD-9-CM administrative databases. J Clin Epidemiol 1992;45:613-9.

14. Hasani A, Pavia D, Rotondetto S, Clarke SW, Spiteri MA, Agnew JE. Effect of oral antibiotics on lung mucociliary clearance during exacerbation of chronic obstructive pulmonary disease. Respir Med 1998;92:442-7.

15. Rosner B. Fundamentals of Biostatistics, 4th edition. Belmont: Wadsworth, 1995:368-9.

16. Schmidt EW, Zimmermann I, Ritzerfeld W, Voss E, Ulmer WT. Controlled prospective study of oral amoxycillin/clavulanate vs ciprofloxacin in acute exacerbations of chronic bronchitis. J Antimicrob Chemother 1989;24(Suppl B):185-93.

17. Wilson R. A vicious circle hypothesis operating during infective exacerbations of chronic bronchitis. Monaldi Arch Chest Dis 1994;49:159-64.

18. Colville A, Knowles M, Large D, George J, Mustchin P. Fluoroquinolones in chronic obstructive pulmonary disease. BMJ 1994;308:1437.

19. Riise GC, Ahlstedt S, Larsson S, et al. Bronchial inflammation in chronic bronchitis assessed by measurement of cell products in bronchial lavage fluid. Thorax 1995;50:360-5.

20. Klein NC. Fluoroquinolones in respiratory infections. Semin Respir Infect 1991;6:131-5.

21. Ball P, Make B. Acute exacerbations of chronic bronchitis: an international comparison. Chest 1998;113(Suppl 3):199S-204S.

22. Kosinski AS, Flanders WD. Evaluating the exposure and disease relationship with adjustment for different types of exposure misclassification: a regression approach. Stat Med 1999;18:2795-808.

23. Murata GH, Gorby MS, Kapsner CO, Chick TW, Halperin AK. A multivariate model for predicting hospital admissions for patients with decompensated chronic obstructive pulmonary disease. Arch Intern Med 1992;152:82-6. 


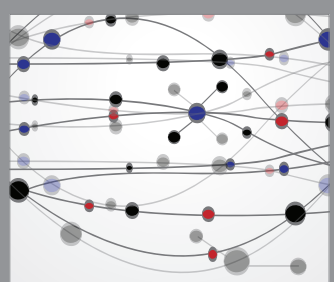

The Scientific World Journal
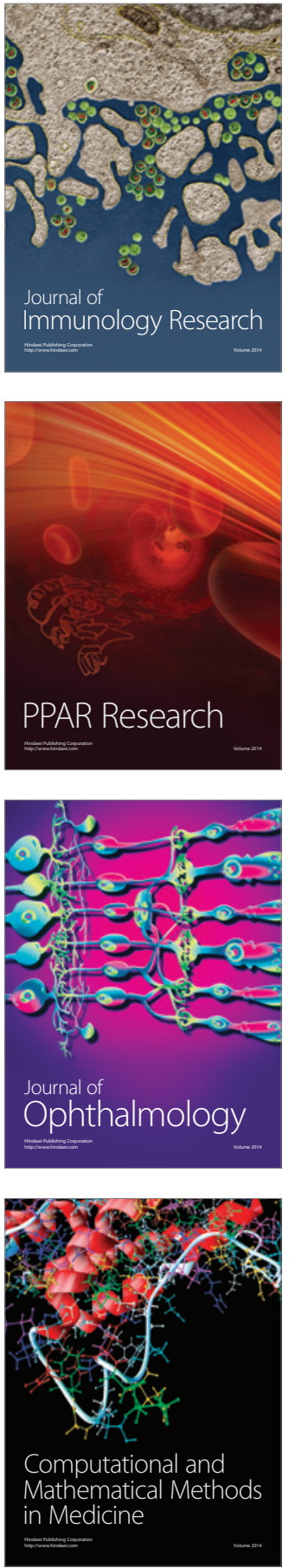

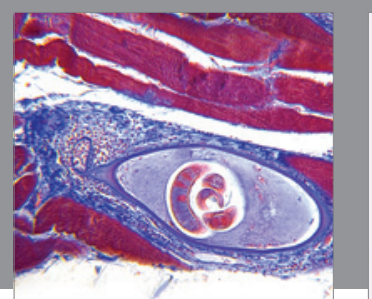

Gastroenterology Research and Practice

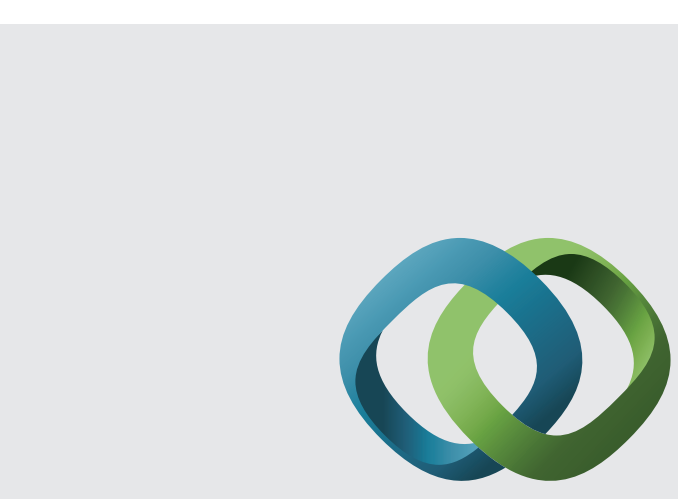

\section{Hindawi}

Submit your manuscripts at

http://www.hindawi.com
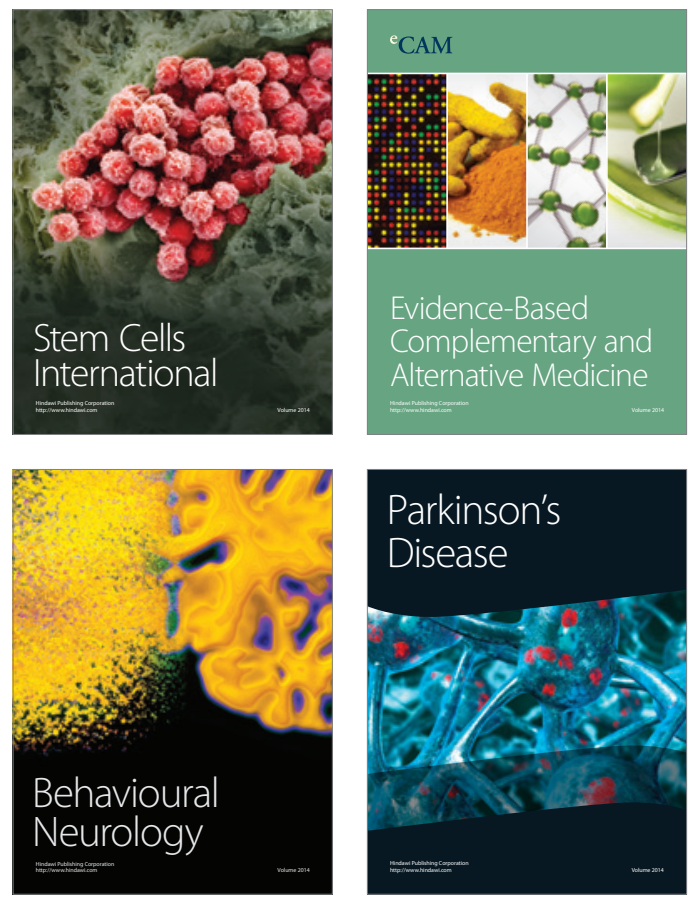
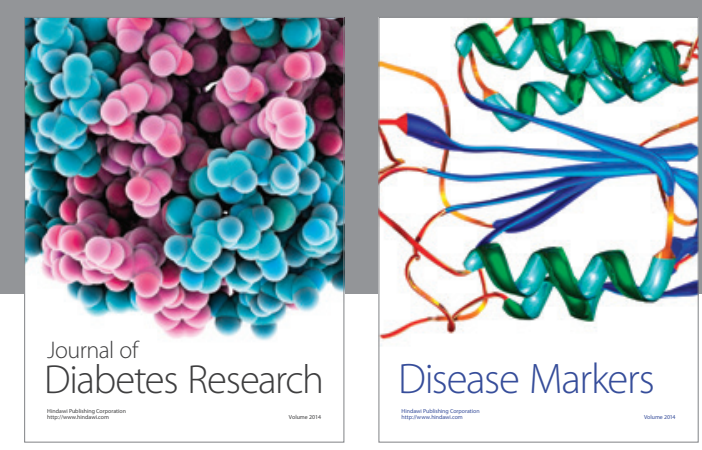

Disease Markers
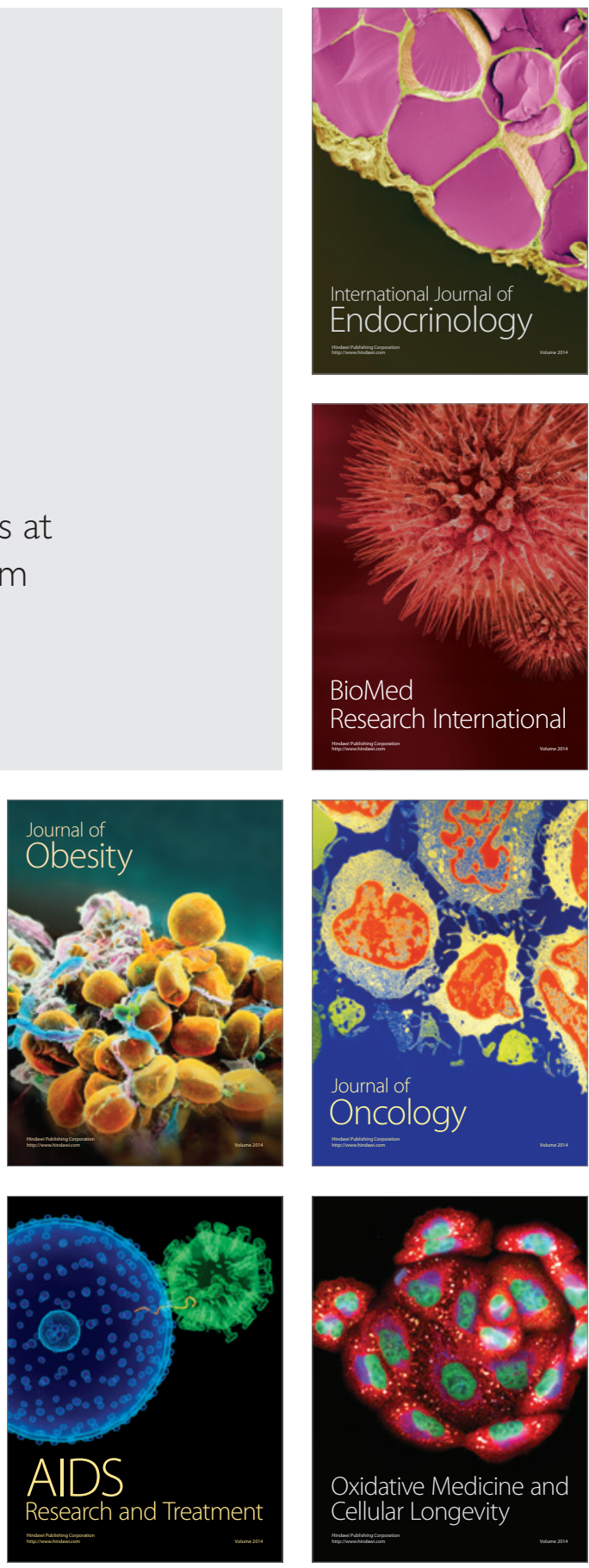